\title{
Effets projetés des mesures de santé publique non pharmacologiques visant à prévenir la recrudescence de la transmission du SRAS-CoV-2
} au Canada

\author{
Victoria Ng PhD, Aamir Fazil MEng, Lisa A. Waddell MSc PhD, Christina Bancej MSc PhD, Patricia Turgeon DMV PhD, \\ Ainsley Otten BEng, Nicole Atchessi PhD, Nicholas H. Ogden BVSc DPhil
}

Citation : CMAJ 2020 September 14;192:E1053-64. doi : 10.1503/cmaj.200990-f; publication hâtive le 10 août 2020

Voir la version anglaise de l'article ici : www.cmaj.ca/lookup/doi/10.1503/cmaj.200990; voir le commentaire connexe (en anglais) ici : www.cmaj.ca/lookup/doi/10.1503/cmaj.201758

\section{RÉSUMÉ}

CONTEXTE : Il faudra prendre des mesures continues contre la transmission communautaire du coronavirus du syndrome respiratoire aigu sévère 2 (SRAS-CoV-2) pour prévenir d'autres vagues d'infection. Nous avons exploré les effets des interventions non pharmacologiques sur la transmission projetée du SRAS-CoV-2 au Canada.

MÉTHODES : Nous avons créé un modèle de la population canadienne à base d'agents intégrant l'âge qui simule les effets des mesures de santé publique, selon leur intensité actuelle et projetée, sur la transmission du SRAS-CoV-2. Les mesures étudiées sont le dépistage et l'isolement des cas, la recherche de contacts et la mise en quarantaine, l'éloignement sanitaire et la fermeture des espaces partagés. Nous avons évalué l'effet des mesures prises individuellement et celui des mesures combinées.

RÉSULTATS : En l'absence de mesures, $64,6 \%$ (intervalle de crédibilité [ $\mathrm{ICr}$ ] à $95 \%$ : 63,9 \%-65,0 \%) des Canadiens contracteraient le SRAS-CoV-2 (taux d'attaque global), et 3,6 \% (ICr à $95 \%$ 2,4 \%-3,8\%) des personnes infectées en mourraient. En poursuivant le dépistage et la recherche de contacts à la même intensité que pendant la période de référence, sans maintenir l'éloignement sanitaire ou refermer certains endroits, le pays connaîtrait un taux d'attaque global de 56,1 \% (ICr à $95 \%$ 0,05 \%-57,1\%); si ces mesures étaient accrues, le taux d'attaque chuterait à 0,4\% (ICr à $95 \%$ 0,03 \%-23,5\%). En combinant ce dernier scénario et le maintien de l'éloignement sanitaire, le taux tomberait à $0,2 \%(\mathrm{ICr}$ à $95 \%$ 0,03\%-1,7\%). Ce scé- nario est le seul qui garderait la demande en soins hospitaliers et intensifs sous la capacité, qui préviendrait presque tous les décès et qui mettrait fin à l'épidémie. La prolongation de la fermeture des écoles aurait un effet minime, mais réduirait la transmission en milieu scolaire. Par contre, la prolongation de la fermeture des lieux de travail et des lieux publics réduirait de manière marquée le taux d'attaque et mettait habituellement ou toujours fin à l'épidémie, selon les différents scénarios simulés.

INTERPRÉTATION : Le contrôle de la transmission du SRAS-CoV-2 passera par l'amélioration et le maintien des mesures, tant communautaires qu'individuelles. Autrement, il y aura une recrudescence de l'épidémie, et un risque de surcharger le système de santé.
L e 11 mars 2020, l'Organisation mondiale de la Santé a officiellement déclaré le statut de pandémie pour la maladie causée par le coronavirus du syndrome respiratoire aigu sévère 2 (SRAS-CoV-2), la maladie à coronavirus 2019 (COVID-19)1,2. En date du 28 juin, on comptait plus de 10 millions de cas confirmés et environ 500000 décès dans 188 pays et territoires ${ }^{3}$. Les personnes infectées par le SRAS-CoV-2 peuvent être asymptomatiques ou présenter des symptômes légers à graves ${ }^{4}$. Les hospitalisations et les décès surviennent surtout chez les personnes âgées ou immunodéprimées ${ }^{5,6}$. Des études semblent montrer que les personnes asymptomatiques peuvent transmettre le virus ${ }^{7}$, et que la contagion précède l'apparition des symptômes ${ }^{8-10}$. Ces caractéristiques, qui diffèrent de celles des autres coronavirus humains, ont posé des défis importants dans le contrôle de la transmission du SRAS-CoV-2 à l'échelle locale et mondiale.

Au départ, la santé publique canadienne misait sur le dépistage chez les voyageurs de retour au pays, afin d'isoler les cas importés. Le premier cas de COVID-19, rapporté le 25 janvier, était effectivement une personne revenant de l'étranger ${ }^{11}$. Les premiers cas détectés en février étaient des voyageurs ou des 
personnes ayant eu des contacts étroits avec des voyageurs ${ }^{12}$. Peu après on remarquait une transmission locale, qui s'est accélérée rapidement en mars, le nombre de cas acquis localement surpassant le nombre de cas liés aux voyages à partir du 15 mars $^{12}$. L'approche de la santé publique est alors passée de l'endiguement à la prévention. Des mesures non pharmacologiques ont été mises en place, comme le dépistage et l'isolement des cas, l'identification des contacts pour les mettre en quarantaine, les mesures d'éloignement sanitaire, notamment la fermeture des espaces partagés, les restrictions de voyage et l'interdiction des grands rassemblements, et d'autres mesures visant à réduire les contacts entre personnes. L'ensemble des provinces et des territoires ont imposé de rigoureuses mesures d'éloignement sanitaire à la mi-mars, qui ont jusqu'à maintenant permis de contrôler l'épidémie ${ }^{13}$. On prévoit toutefois qu'en l'absence de mesures continues de prévention de la transmission communautaire, d'autres vagues d'infection suivront. Puisque les mesures utilisées pour endiguer la COVID19 sont sans précédent et que leur efficacité demeure inconnue, la modélisation de leurs effets probables dans une variété de scénarios peut faciliter la prise de décisions ${ }^{14-16}$.

La dynamique de transmission des maladies infectieuses est habituellement modélisée à l'aide de modèles compartimentaux à l'échelle populationnelle ${ }^{17,18}$. Les modèles à base d'agents, quant à eux, fonctionnent avec des simulations par ordinateur faisant intervenir des agents qui représentent des personnes, des endroits ou des objets. Ces modèles sont stochastiques, et les agents sont programmés pour interagir entre eux dans l'environnement défini. Comme les agents peuvent avoir des caractéristiques uniques et qu'une hétérogénéité est possible, ce type de modèle convient à la simulation d'événements qui arrivent par hasard, par exemple la contagion à l'apparition des premiers symptômes et l'élimination du virus se produit dans les derniers stades de la maladie. Les modèles à base d'agents sont particulièrement adaptés à l'étude de l'efficacité des mesures de prévention, puisque celles-ci dépendent largement de la structure communautaire et de la dynamique dans la population ${ }^{19,20}$. Nous présentons ici un modèle à base d'agents conçu à l'Agence de la santé publique du Canada, qui sert à prédire la transmission du SRAS-CoV-2 selon l'imposition de différentes mesures au Canada.

\section{Méthodes}

Les détails techniques du modèle, dont les paramètres dérivés d'études publiées et de données canadiennes, sont présentés à l'annexe 1 (accessible ici : www.cmaj.ca/lookup/doi/10.1503/ cmaj.200990/tab-related-content). Notre modèle a été créé à l'aide d'AnyLogic Professional 8.5.2 (The AnyLogic Company). De multiples éclosions étant en cours au Canada, le modèle donne une estimation générale de la situation et ne tient pas compte de la dispersion géographique des cas.

\section{Mesures et scénarios du modèle}

Le modèle intègre 4 mesures (dépistage et isolement des cas, recherche de contacts et mise en quarantaine, éloignement sanitaire permettant de réduire le taux de contacts quotidiens, et fermeture des espaces partagés), qu'il étudie de manière séparée et combinée (tableau 1). Nous avons comparé l'efficacité de chaque mesure à celle de l'absence de mesures pour évaluer la progression libre de la COVID-19 dans une population naïve. Nous avons modélisé les mesures séparément pour connaître leur effet indépendant, et nous avons estimé leur intensité actuelle pour générer des données de référence. Ces données décrivent la situation au Canada pour la période du 7 février au 10 mai 2020, date où

\section{Tableau 1 : Mesures non pharmacologiques modélisées}

Mesure

Dépistage et isolement des cas

Recherche de contacts et mise en quarantaine

Éloignement sanitaire

Fermeture des espaces partagés

\section{Effets}

Dépistage visant la détection des cas symptomatiques et l'isolement des personnes testées positives chez elles pendant 14 jours, et donc la réduction de la transmission communautaire. Une certaine proportion des membres du ménage s'isolent aussi, sauf s'ils ont déjà été infectés et donc qu'ils sont immunisés.

Au moment où les agents commencent à avoir des symptômes légers, ils sont déjà contagieux depuis 1 à 3 jours, mais leur isolement à ce moment prévient 3 à 7 jours supplémentaires de transmission communautaire potentielle. Les agents isolés peuvent toujours infecter ceux qui vivent avec eux, mais à un taux de contacts estimé de $50 \%$, car nous présumons que les personnes malades imposeront une forme d'éloignement sanitaire dans le ménage.

Recherche de contacts visant à repérer les personnes exposées infectées, mais qui ne sont pas encore contagieuses. Cette mesure est l'une des plus efficaces, parce qu'un agent mis en quarantaine pendant 14 jours après l'exposition ne transmettra à aucun moment la maladie.

Réduit le nombre de contacts par jour; peut être appliqué à toute la population ou à des groupes d'âge ciblés. N'a été imposé qu'à l'extérieur du ménage.

Fermeture des écoles, des lieux de travail et des lieux publics définie soit par une proportion (100\% des écoles) soit par un seuil (lieux de travail comptant 50 agents ou plus). Les agents assignés à un espace partagé fermé restent à la maison jusqu'à la réouverture.
Agents concernés

Symptômes légers*

Exposés

Tous les états de santé

S.O.

Remarque : S.O. = sans objet.

*On suppose que les personnes présentant des symptômes graves sont trop malades pour sortir; le dépistage et l'isolement s'appliquent donc uniquement aux agents présentant des symptômes légers. 
les espaces partagés ont commencé à rouvrir. Nous estimons que pendant la période de référence, environ $20 \%$ des cas symptomatiques ont été détectés par dépistage et isolés pour le reste de la période de contagion ${ }^{21} ; 50 \%$ des membres des ménages des cas identifiés se sont aussi isolés (estimation); 50 \% des personnes exposées ont été repérées par la recherche de contacts et mises en quarantaine avant d'être contagieuses (estimation); $100 \%$ des écoles, $40 \%$ des lieux de travail et $50 \%$ des lieux publics ont été fermés du 16 mars au 10 mai 22,23 ; et l'éloignement sanitaire à l'extérieur du domicile a fait diminuer le taux de contacts de $20 \%$ supplémentaires ${ }^{24,25}$. Les données de référence ont été validées selon le nombre de cas acquis localement au Canada (annexe 1, figure S2).

À partir de la situation de référence, nous avons modélisé 4 scénarios pour la période du 11 mai 2020 au 7 janvier 2022, qui illustrent des trajectoires potentielles de transmission du SRASCoV-2 au Canada selon l'intensité des mesures mises en place pour compenser la réouverture des espaces partagés (figure 1). Les scénarios sont les suivants : mesures de prévention minimales (dépistage et isolement des cas, recherche de contacts et mise en quarantaine à la même intensité que durant la période de référence, et abandon de l'éloignement sanitaire); maintien de l'éloignement sanitaire; dépistage et recherche de contacts accrus, et mesures combinées (maintien de l'éloignement sanitaire, et dépistage et recherche de contacts accrus) (tableau 2).

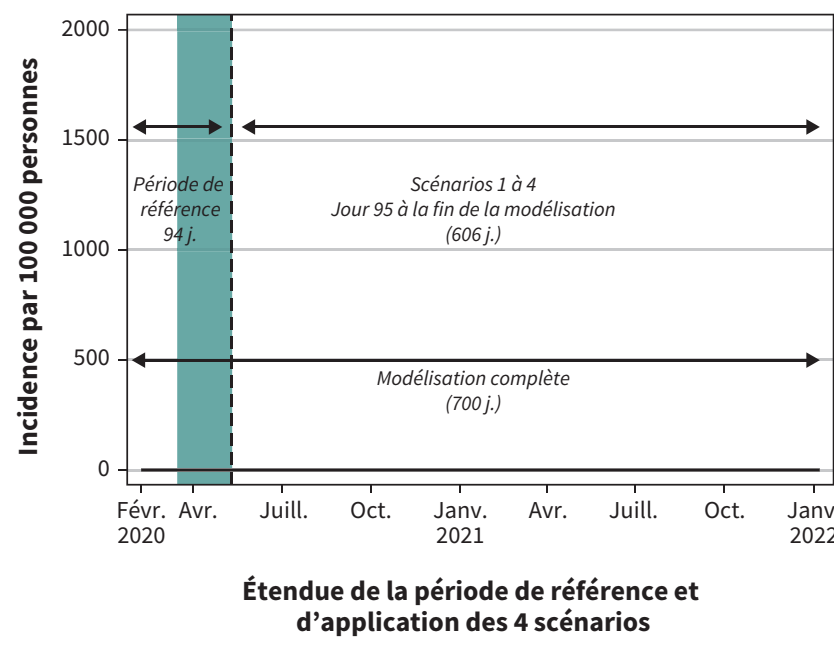

Figure 1: Les données de référence sont appliquées aux 4 scénarios pour les 94 premiers jours de la simulation. À partir du $95^{\mathrm{e}}$ jour (11 mai 2020), une autre combinaison de mesures de santé publique est appliquée dans chaque scénario. Remarque : La simulation complète s'étend sur 700 jours, du 7 février 2020 au 7 janvier 2022. La bande verte représente les 8 semaines de fermeture des espaces partagés (lieux de travail, écoles, lieux publics - centres commerciaux, bibliothèques, etc.) pendant la période de référence, du 16 mars au 10 mai 2020.

Tableau 2 : Résumé de la situation canadienne de référence et intensité des mesures de santé publique mises en place dans les 4 scénarios à l'étude

Scénarios et intensité des mesures (11 mai 2020 au 7 janvier 2022)

\begin{tabular}{|c|c|c|c|c|c|}
\hline Mesure & $\begin{array}{l}\text { Situation de référence } \\
\text { ( } 7 \text { février au } \\
10 \text { mai 2020) }\end{array}$ & $\begin{array}{l}\text { Mesures minimales } \\
\text { (aucun changement aux } \\
\text { mesures après la } \\
\text { réouverture, et } \\
\text { non-maintien de } \\
\text { l'éloignement sanitaire) }\end{array}$ & $\begin{array}{l}\text { Maintien de } \\
\text { l'éloignement } \\
\text { sanitaire }\end{array}$ & $\begin{array}{l}\text { Dépistage et } \\
\text { recherche de } \\
\text { contacts accrus }\end{array}$ & $\begin{array}{l}\text { Mesures } \\
\text { combinées }\end{array}$ \\
\hline $\begin{array}{l}\text { Dépistage et } \\
\text { isolement des cas }\end{array}$ & $\begin{array}{c}20 \% \text { des cas sont } \\
\text { dépistés et isolés (50\% } \\
\text { des membres du ménage } \\
\text { s'isolent aussi) }\end{array}$ & $\begin{array}{l}20 \% \text { des cas sont } \\
\text { dépistés et isolés ( } 50 \% \\
\text { des membres du } \\
\text { ménage s'isolent aussi) }\end{array}$ & $\begin{array}{c}20 \% \text { des cas sont } \\
\text { dépistés et isolés } \\
\text { (50\% des membres } \\
\text { du ménage s'isolent } \\
\text { aussi) }\end{array}$ & $\begin{array}{c}50 \% \text { des cas sont } \\
\text { dépistés et isolés* } \\
\text { (50\% des membres } \\
\text { du ménage s'isolent } \\
\text { aussi) }\end{array}$ & $\begin{array}{c}50 \% \text { des cas sont } \\
\text { dépistés et isolés* } \\
\text { (50\% des membres } \\
\text { du ménage } \\
\text { s'isolent aussi) }\end{array}$ \\
\hline $\begin{array}{l}\text { Recherche de } \\
\text { contacts et mise en } \\
\text { quarantaine }\end{array}$ & $\begin{array}{c}50 \% \text { des contacts des } \\
20 \% \text { de cas dépistés } \\
\text { sont retracés et mis en } \\
\text { quarantaine }\end{array}$ & $\begin{array}{c}50 \% \text { des contacts des } \\
20 \% \text { de cas dépistés } \\
\text { sont retracés et mis en } \\
\text { quarantaine }\end{array}$ & $\begin{array}{l}50 \% \text { des contacts } \\
\text { des } 20 \% \text { de cas } \\
\text { dépistés sont } \\
\text { retracés et mis en } \\
\text { quarantaine }\end{array}$ & $\begin{array}{l}100 \% \text { des contacts } \\
\text { des } 50 \% \text { de cas } \\
\text { dépistés sont } \\
\text { retracés et mis en } \\
\text { quarantaine }\end{array}$ & $\begin{array}{l}100 \% \text { des contacts } \\
\text { des } 50 \% \text { de cas } \\
\text { dépistés sont } \\
\text { retracés et mis en } \\
\text { quarantaine }\end{array}$ \\
\hline $\begin{array}{l}\text { Éloignement } \\
\text { sanitaire (réduction } \\
\text { du taux de contacts } \\
\text { quotidiens) }\end{array}$ & $\begin{array}{c}\text { Réduction de } 20 \% \text { du } \\
\text { taux de contacts pendant } \\
8 \text { semaines (du } 16 \text { mars } \\
\text { au } 10 \text { mai 2020) }\end{array}$ & S.O. & $\begin{array}{l}\text { Maintien de la } \\
\text { réduction de } 20 \% \\
\text { du taux de } \\
\text { contacts* }\end{array}$ & S.o. & $\begin{array}{l}\text { Maintien de la } \\
\text { réduction de } 20 \% \\
\text { du taux de } \\
\text { contacts* }\end{array}$ \\
\hline $\begin{array}{l}\text { Fermeture des } \\
\text { espaces partagés }\end{array}$ & $\begin{array}{c}100 \% \text { des écoles, } 40 \% \\
\text { des lieux de travail et } \\
50 \% \text { des lieux publics } \\
\text { sont fermés pendant } \\
8 \text { semaines (du } 16 \text { mars } \\
\text { au } 10 \text { mai } 2020 \text { ) }\end{array}$ & S.O. & S.O. & S.O. & S.O. \\
\hline
\end{tabular}


Tous ont été créés à partir de cibles canadiennes jugées réalistes compte tenu des mesures en place. Puis nous nous sommes servis des scénarios pour comparer et explorer les effets de la fermeture prolongée des écoles et des espaces partagés. Nous avons modélisé des fermetures qui se prolongeraient jusqu'en septembre 2020. Lorsque nous supposions une prolongation de la fermeture des écoles, nous levions la fermeture des lieux de travail et des lieux publics, et vice versa. Comme les écoles sont de toute façon fermées pendant l'été, nous avons pu faire une comparaison pour une même période.

Tableau 3 : Résumé des résultats de la modélisation pour les 4 scénarios à l'étude*

\section{Variable}

Taux d'attaque global, \% (ICr à $95 \%)$

Taux d'attaque clinique, \% (ICr à $95 \%)$

Taux de cas asymptomatiques, \% (ICr à $95 \%)$

Proportion de cas asymptomatique sur le nombre total de cas, \% (ICr à $95 \%)$

Cas cliniques légers (pas hospitalisés), \% (ICrà $95 \%)$

Cas cliniques hospitalisés (y compris aux soins intensifs), \% (ICr à $95 \%)$

Cas cliniques admis aux soins intensifs, \% (ICr à $95 \%)$

Cas hospitalisés admis aux soins intensifs, $\%$ (ICr à $95 \%)$

Taux de mortalité parmi les cas cliniques, \% (ICr à $95 \%)$

Total des cas (cliniques et asymptomatiques) par 100000 personnes, médiane (ICr à $95 \%)$

Total des cas cliniques par 100000 personnes, médiane (ICr à $95 \%)$

Total des cas asymptomatiques par 100000 personnes, médiane (ICr à $95 \%$ )

Total des cas hospitalisés par

100000 personnes, médiane (ICr à $95 \%)$

Total des cas admis aux soins intensifs par 100000 personnes, médiane (ICr à $95 \%)$

Total des décès par 100000 personnes, médiane (ICr à $95 \%)$

Infections acquises à l'école, \% (ICr à $95 \%)$

Infections acquises au travail, \% (ICr à $95 \%$ )

Infections acquises dans des lieux publics, $\%$ (ICr à $95 \%)$

Infections acquises à domicile, \% (ICr à $95 \%$ )

Nombre d'infections acquises à l'école par

100000 personnes, médiane (ICr à 95 \%)

Nombre d'infections acquises au travail par 100000 personnes, médiane (ICr à $95 \%$ )

Nombre d'infections acquises dans des lieux publics, médiane (ICr à $95 \%)$

Nombre d'infections acquises à domicile par 100000 personnes (ICr à $95 \%)$

\section{Mesures minimales}

$56,1(0,05-57,1)$

$34,5(0,03-35,1)$

$21,6(0,01-22,1)$

$38,5(26,7-42,5)$

$89,1(4,1-21,2)$

$10,9(4,1-21,2)$

$2,8(0,0-7,9)$

$25,4(0,0-50,0)$

$3,2(0,0-3,5)$

$56148(45-57068)$

$34463(31-35087)$

$21615(12-22$ 101)

3747 (2-3903)

$950(0-1031)$

$1113(0-1208)$

$9,1(1,7-9,7)$

$14,2(9,1-20,5)$

$25,4(14,3--28,2)$

$51,2(50,8-68,2)$

$5134(1-5336)$

7947 (4--8239)

14225 (6-14 694)

$28659(24-29137)$
$16152(14-16881)$

Maintien de

l'éloignement

sanitaire

$41,6(0,04-43,4)$

$25,4(0,03-26,6)$

$16,2(0,01-16,9)$

$38,8(28,6-40,2)$

$89,5(84,0-94,9)$

$10,5(5,1-16,0)$

$2,7(0,0-5,9)$

$25,4(0,0-50,0)$

$2,9(0,0-4,8)$

$41579(44-43455)$

$25413(30-26626)$

$2661(3-2824)$

$665(0-748)$

$739(0-830)$

$8,9(0,0-9,4)$

$13,7(5,3-19,5)$

$24,0(12,3-27,2)$

$53,3(52,9-64,9)$

$3759(0-3920)$

$5655(2-6000)$

9941 (6-10 503)

$22176(23-23080)$
Dépistage et

recherche de

contacts accrus

$0,36(0,03-23,5)$

$0,23(0,02-14,3)$

$0,13(0,01-9,2)$

$38,9(24,1-40,5)$

$89,7(80,0-93,0)$

$90,0(60,9-93,6)$

$10,3(7,0-20,0)$

$10,0(6,5-39,1)$

$2,6(0,0-5,0)$

$2,7(0,0-6,2)$

$25,4(0,0-40,0)$

$24,1(0,0-57,1)$

$1,7(0,0-4,0)$

$1,4(0,0--5,3)$

247 (28-1679)

157 (20-991)

$94(8-688)$

$16(4-105)$

$4(0-28)$

$2(0-13)$

$4,8(0,0-10,8)$

$13,3(5,8-19,4)$

$13,1(5,8-25,8)$

$23,2(11,1-27,8)$

$22,4(13,6-30,3)$

$56,2(53,1-69,2)$

$18(0-2365)$

$58,4(51,6-69,7)$

11 (0-159)

$33(3-217)$

$43(2-3107)$

$56(3-371)$

$83(2-5511)$

142 (15-942)

Remarque : $\mathrm{ICr}=$ intervalle de crédibilité.

*Le tableau donne la médiane pour 50 simulations et les valeurs du 2,5e centile et du $97,5^{e}$ centile, qui délimitent l'ICrà $95 \%$. Un ICr important indique un résultat dichotomique entre les simulations (p. ex. poursuite ou fin de l'épidémie). La médiane correspond au résultat le plus probable des 50 simulations. 


\section{Variables et analyses statistiques}

Avec le modèle, nous avons produit un cumulatif et un décompte quotidiens des cas pour chaque groupe d'âge et pour chaque état de santé. Parmi les variables évaluées, citons le taux d'attaque global (total des nouveaux cas divisé par la population totale), le taux d'attaque clinique, le taux de cas asymptomatiques, le nombre d'hospitalisations, le nombre d'admissions aux soins intensifs et le taux de mortalité par groupes d'âge. Nous avons aussi construit des courbes épidémiques représentant l'incidence et la prévalence quotidiennes de la maladie par 100000 personnes. Les résultats stochastiques sont présentés comme les médianes de 50 essais (simulations) par scénario, et les valeurs du $2,5^{\mathrm{e}}$ et du $97,5^{\mathrm{e}}$ centiles, comme l'intervalle de crédibilité $(\mathrm{ICr})$ à $95 \%$. Le paramètre de transmission a été adapté aux données canadiennes, ce qui donne un taux de reproduction $\left(\mathrm{R}_{0}\right.$; nombre moyen de personnes infectées par une même personne) de 2,7. Nous avons également réalisé des analyses de sensibilité, afin de voir l'effet de la variation entre 2,0 et 3,0 du $R_{0}$ pour chacun des 4 scénarios. Enfin, nous avons fait nos analyses statistiques et créé les graphiques à l'aide de Stata 16 (StataCorp).

\section{Approbation éthique}

Aucune approbation éthique n'était nécessaire pour cette étude : toutes les données proviennent d'articles publiés ou sont accessibles au public sous forme agrégée.

\section{Résultats}

Le taux d'attaque global de la population pour la transmission communautaire en l'absence de mesures de prévention serait de $64,6 \%(\mathrm{ICr}$ à $95 \%$ 63,9 \%-65,0\%) et le taux de mortalité, de 3,6\% (ICr à $95 \%$ 2,4 \%-3,8 \%) (annexe 1, figure S3). Nous avons modélisé les mesures séparément pour connaître leur efficacité lorsqu'elles sont utilisées seules. La mesure la plus efficace serait la fermeture partielle des espaces partagés (taux d'attaque global 7,6 \%, ICr à
A

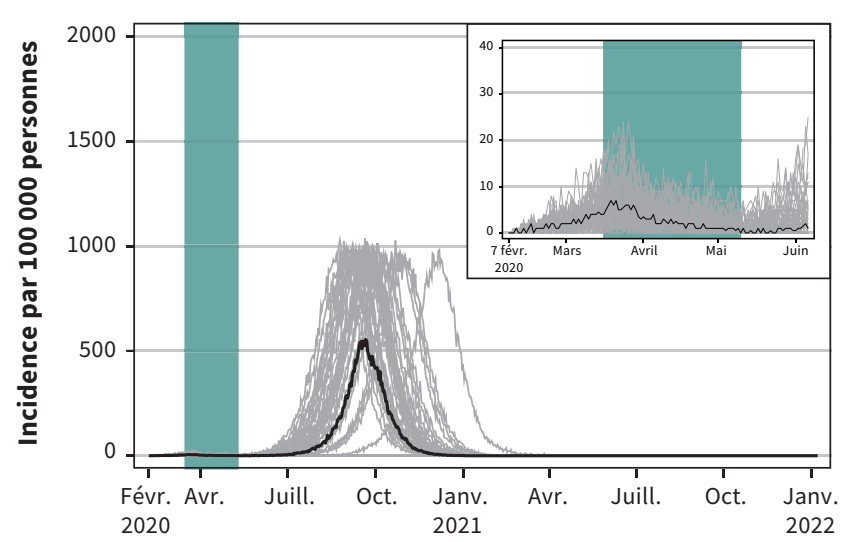

B

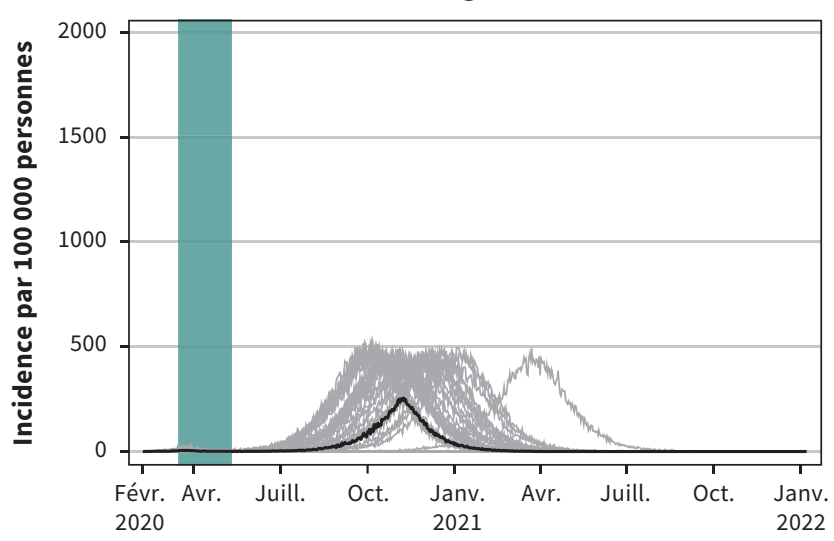

C

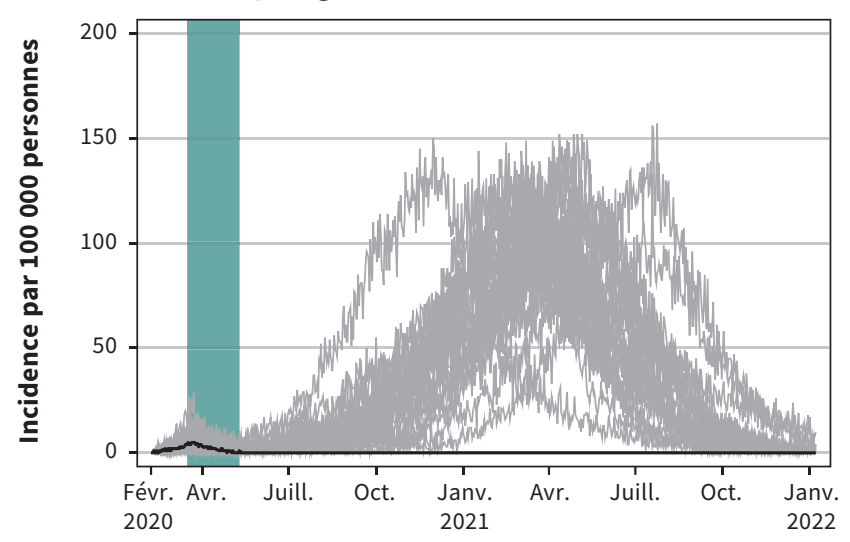

D

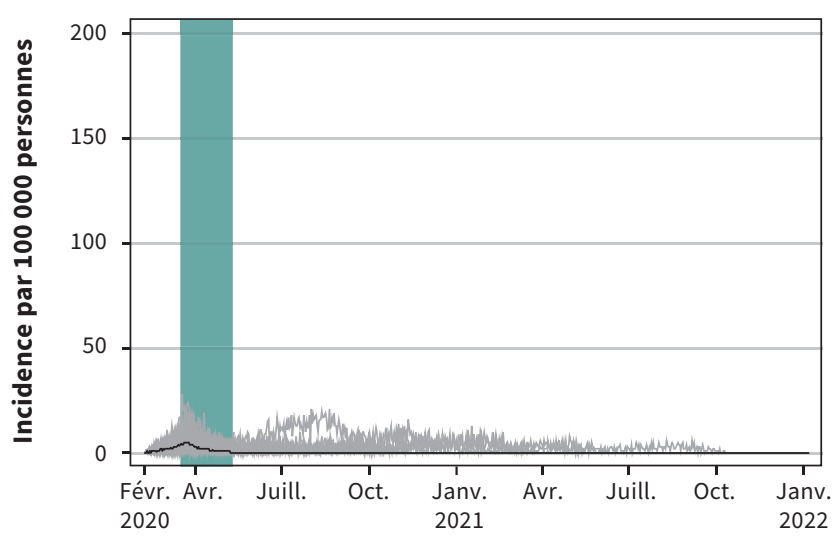

Figure 2: Courbes épidémiques projetées du nombre quotidien de cas par 100000 personnes pour les 4 scénarios. Dans chaque scénario, les mesures de santé publique s'intensifient graduellement suivant la réouverture des espaces partagés. Remarque : La bande verte représente la période du 16 mars au 10 mai 2020, soit la période de fermeture. La comparaison des 4 scénarios montre à quel point le retrait de certaines mesures, avec le maintien ou l'accroissement d'autres mesures, entraînerait une perte de contrôle de l'épidémie au Canada alors que la transmission communautaire se poursuit. La ligne noire représente la médiane. Chaque ligne grise représente 1 simulation; il y en a 50 par scénario. Remarque : L'échelle de l'axe des y est 10 fois plus petite pour le scénario de dépistage et de recherche de contacts accrus et le scénario des mesures combinées que pour les 2 autres scénarios. 
$95 \%$ 0,36 \%-13,2 \%), suivie par le maintien de l'éloignement sanitaire (taux d'attaque global 54,0 \%, ICr à $95 \%$ 53,0 \%-54,7\%), le dépistage et l'isolement de $20 \%$ des cas et le co-isolement de $50 \%$ des membres du ménage (taux d'attaque global 59,3\%, ICr à $95 \%$ 0,04\%-60,0\%) et enfin l'identification et la mise en quarantaine de $50 \%$ des contacts (taux d'attaque global 62,5\%, ICr à $95 \%$ $62,0 \%-63,3 \%$ ) (annexe 1, figures S4-S7). La seule mesure qui permettrait de mettre fin à l'épidémie à elle seule serait la fermeture partielle des espaces partagés (mais après 18 mois, et il est probablement irréaliste d'appliquer aussi longtemps une mesure aussi restrictive), alors que toutes les autres mesures différeraient l'épidémie. Aux fins de comparaison, nous avons aussi modélisé les mesures combinées de la période de référence (à l'exception de la fermeture des espaces partagés); nous avons obtenu un taux d'attaque global de 42,3\% ( $\mathrm{ICr}$ à $95 \%$ 0,03\%-43,3\%), soit une diminution de $22,3 \%$ comparativement à une diminution de $17,8 \%$ pour les mêmes mesures appliquées séparément (annexe 1, figure S8; données supplémentaires disponibles sur demande auprès des auteurs).

A Mesures minimales
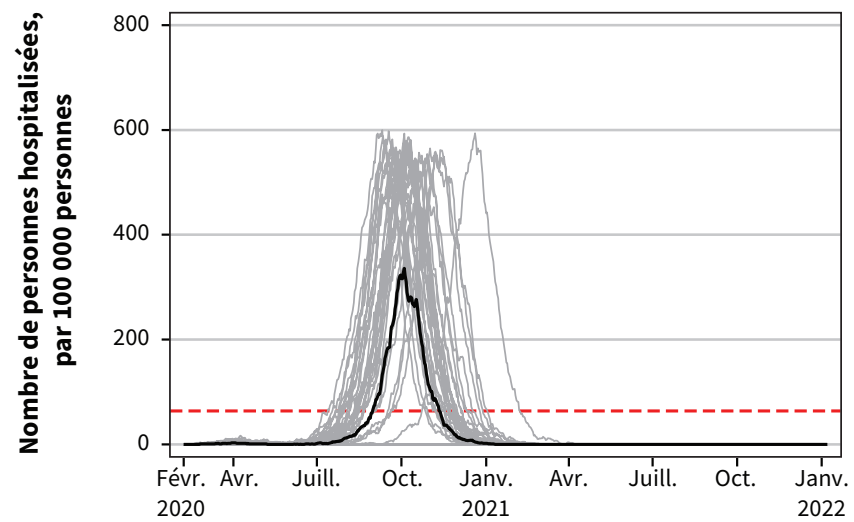

C Dépistage et recherche de contacts accrus
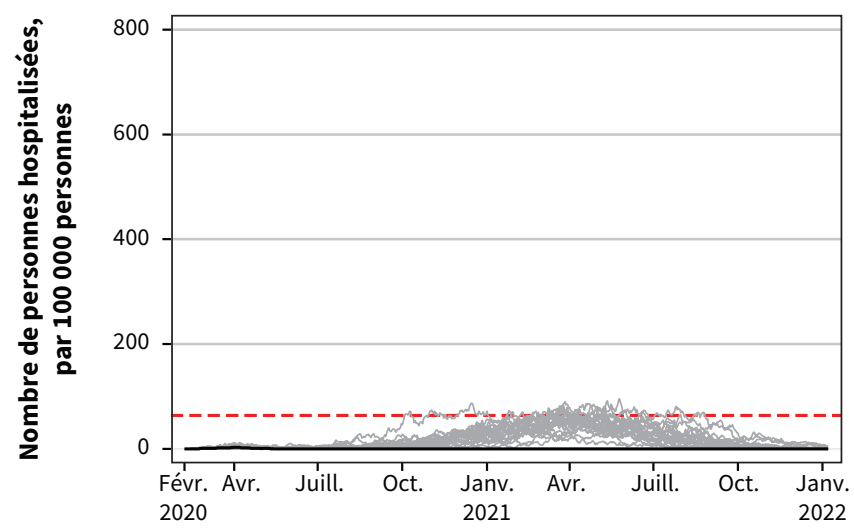

Le taux d'attaque global pour le scénario des mesures minimales est de 56,1\% (ICr à $95 \%$ 0,05 \%-57,1\%) (tableau 3). Le taux d'attaque global le plus élevé, qui s'explique un pourcentage de personnes asymptomatiques élevé ( $\approx 50 \%$ ), est observé chez les 10 à 19 ans, le taux d'attaque clinique le plus élevé, chez les 20 à 54 ans, les taux d'hospitalisation et de mortalité les plus élevés, chez les personnes de 75 ans et plus, et le taux d'admission aux soins intensifs le plus élevé, chez les 65 à 84 ans (parce que la plupart des personnes de 85 ans et plus décèdent avant de se rendre) (données disponibles sur demande auprès des auteurs). Le scénario des mesures minimales, qui correspond au dépistage et à la recherche de contacts à la même intensité qu'à la période de référence a permis de réduire efficacement le taux d'attaque global, mais ne suffisait pas à endiguer l'épidémie ou à contrôler une recrudescence de l'épidémie à la levée des mesures restrictives (fermetures) (figure 2).

Le scénario des mesures minimales, accompagné du maintien de l'éloignement sanitaire, donne un taux d'attaque global réduit à 41,6\% (ICr à $95 \%$ 0,04\%-43,4\%). En comparaison, le
B Maintien de l'éloignement sanitaire

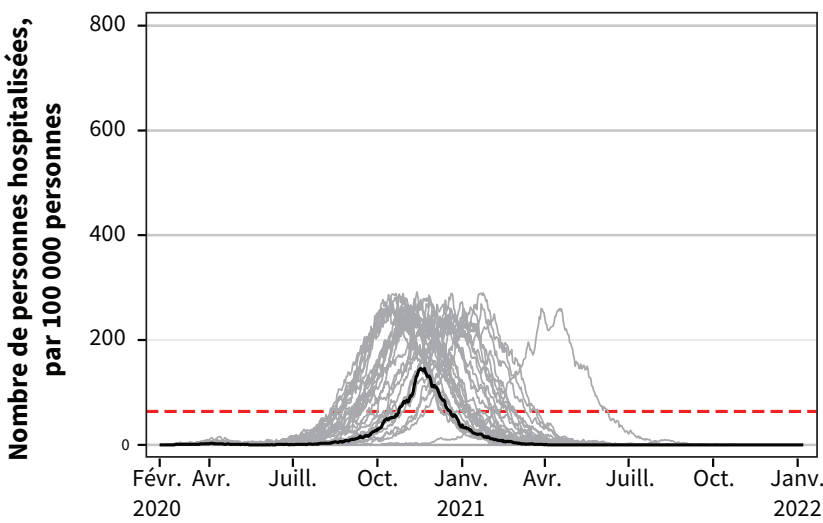

D

Mesures combinées

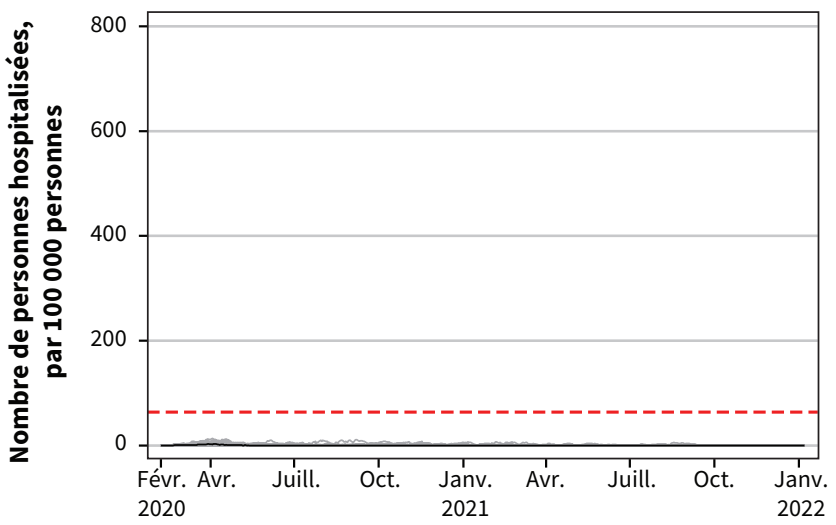

Figure 3: Projections de la demande en lits d'hôpital, montrant le total quotidien de cas hospitalisés par 100000 personnes pour les 4 scénarios. Remarque : Le nombre de personnes hospitalisées comprend les patients admis en soins généraux et admis à l'hôpital avant ou après un passage aux soins intensifs en raison de la maladie à coronavirus 2019. La capacité maximale des hôpitaux canadiens est représentée par la ligne traitillée rouge. La ligne noire représente la médiane. Chaque ligne grise représente 1 simulation; il y en a 50 par scénario. 
scénario du dépistage et de la recherche de contacts accrus fait tomber le taux d'attaque global à $0,4 \%(\mathrm{ICr}$ à $95 \%$ 0,03\%-23,5\%). Dans le dernier scénario, qui combine ces mesures et l'éloignement sanitaire, le taux d'attaque global est de $0,2 \%$ (ICr à $95 \%$ 0,03\%-1,7\%). Toutefois, seul le scénario des mesures combinées éradique l'épidémie, malgré certaines simulations indiquant qu'elle pourrait ne pas être contrôlée avant l'automne 2021 (figure 2).

Les effets comparés des 4 scénarios sur les cas cliniques, les hospitalisations et les admissions aux soins intensifs vont dans le même sens que les effets sur le taux d'attaque global (tableau 3). Nous sommes arrivés à un total de 1113 morts par 100000 personnes (ICr à $95 \% 0-1208)$ pour les mesures minimales, $739(\mathrm{ICr}$ à $95 \% 0-830)$ pour le maintien de l'éloignement sanitaire, $4 \mathrm{ICr}$ à $95 \%$ 0-296) pour le dépistage et la recherche de contacts accrus et 2 ( $\mathrm{Cr}$ à $95 \%$ 0-13) pour les mesures combinées. Comparativement à l'absence de mesures de santé publique, les mesures minimales préviendraient 5312 cas cliniques, 798 hospitalisations et 320 décès par 100000 personnes, alors que les mesures combinées préviendraient 39618 cas cliniques,
4529 hospitalisations et 1431 décès par 100000 personnes (données disponibles sur demande auprès des auteurs). Dans les scénarios des mesures minimales et du maintien de l'éloignement sanitaire, la demande de soins hospitaliers et intensifs excéderait la capacité maximale (figures 3 et 4). Dans celui du dépistage et de la recherche de contacts accrus, la demande reste sous la capacité dans la plupart des simulations, mais la dépasse dans certaines. Seules les mesures combinées permettent dans tous les cas de rester sous la capacité maximale des hôpitaux et des services de soins intensifs.

La prolongation de la fermeture des écoles dans les scénarios des mesures minimales et de maintien de l'éloignement sanitaire réduit très peu le taux d'attaque global par rapport aux scénarios seuls (tableau 4) et ne fait qu'allonger l'épidémie (figure 5). Cela dit, elle préviendrait 924 cas cliniques, 95 hospitalisations et 26 décès par 100000 personnes (tableaux 3 et 4) comparativement aux mesures minimales seules, et 351 cas cliniques, 71 hospitalisations et 28 décès par 100000 personnes comparativement au seul maintien de l'éloignement sanitaire. En outre, elle réduirait le nombre d'infections acquises dans les
A

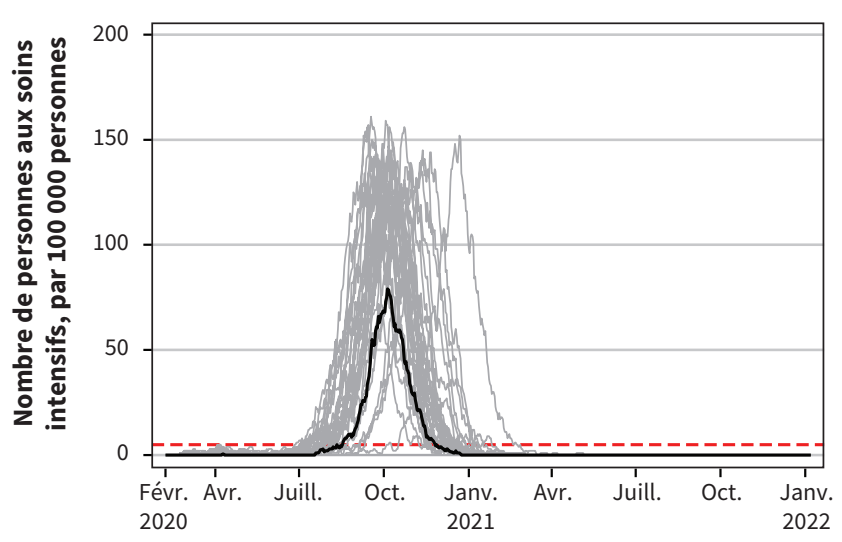

B

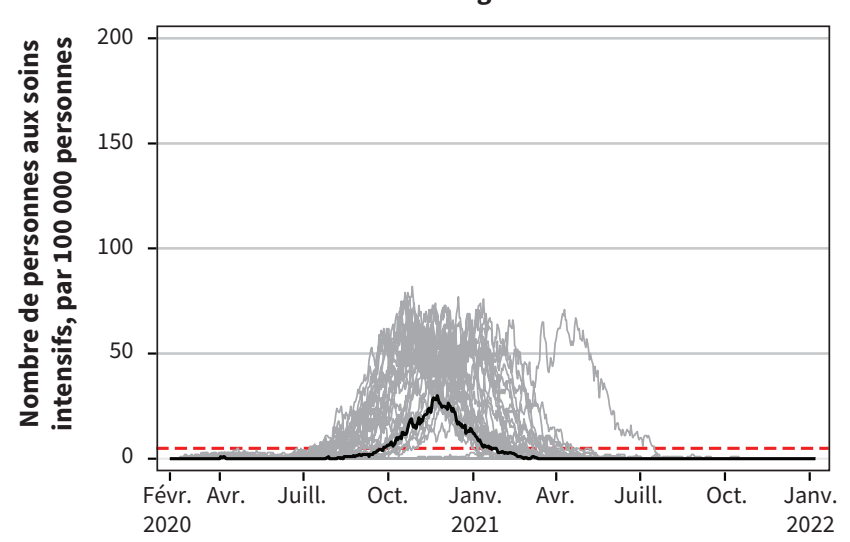

C Dépistage et recherche de contacts accrus

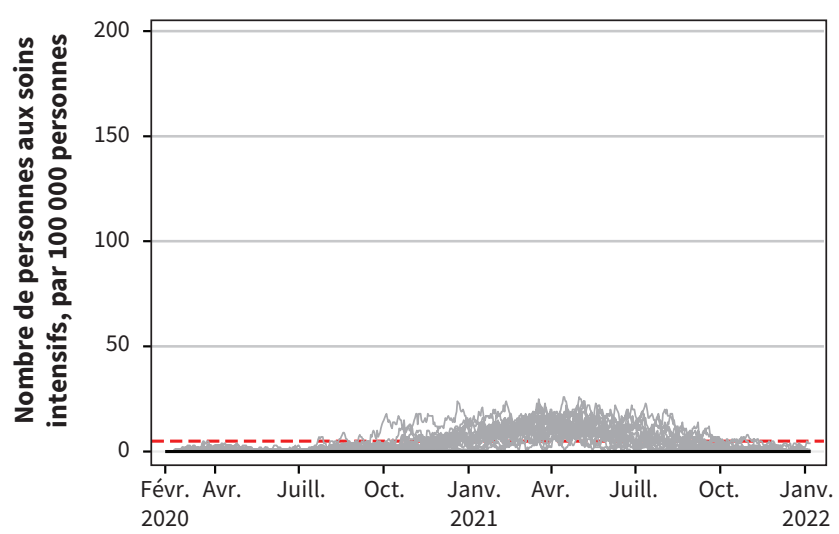

D

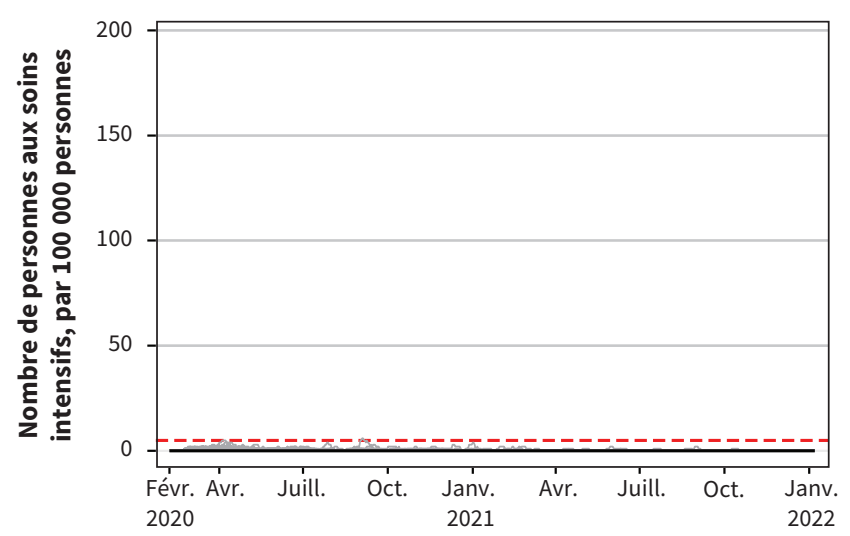

Figure 4: Projections de la demande en lits de soins intensifs, montrant le total quotidien de personnes aux soins intensifs par 100000 personnes pour les 4 scénarios. Remarque : La capacité maximale des services de soins intensifs canadiens est représentée par la ligne traitillée rouge. La ligne noire représente la médiane. Chaque ligne grise représente 1 simulation; il y en a 50 par scénario. 
Tableau 4 : Résumé des résultats de la modélisation pour les 4 scénarios à l'étude, avec prolongation de la fermeture des écoles

\section{Variable}

Taux d'attaque global, \% (ICr à $95 \%)$

Taux d'attaque clinique, \% (ICr à $95 \%$ )

Taux de cas asymptomatiques, \% (ICr à $95 \%)$

Proportion de cas asymptomatique sur le nombre total de cas, \% (ICr à $95 \%)$

Cas cliniques légers (pas hospitalisés), \% (ICr à $95 \%$ )

Cas cliniques hospitalisés (y compris aux soins intensifs), \% (ICr à $95 \%$ )

Cas cliniques admis aux soins intensifs, \% (ICr à 95\%)

Cas hospitalisés admis aux soins intensifs,

$\%$ (ICr à $95 \%)$

Taux de mortalité parmi les cas cliniques, \% (ICr à $95 \%)$

Total des cas (cliniques et asymptomatiques) par 100000 personnes, médiane (ICr à $95 \%$ )

Total des cas cliniques par 100000 personnes, médiane ( $\mathrm{ICr}$ à $95 \%)$

Total des cas asymptomatiques par 100000 personnes, médiane (ICr à 95 \%)

Total des cas hospitalisés par 100000 personnes, médiane (ICr à $95 \%)$

Total des cas admis aux soins intensifs par 100000 personnes, médiane (ICr à $95 \%)$

Total des décès par 100000 personnes, médiane (ICr à $95 \%)$

Infections acquises à l'école, \% (ICr à $95 \%$ )

Infections acquises au travail, \% (ICr à $95 \%$ )

Infections acquises dans des lieux publics, \% (ICr à $95 \%)$

Infections acquises à domicile, \% (ICr à $95 \%)$

Nombre d'infections acquises à l'école par 100000 personnes, médiane (ICr à $95 \%$ )

Nombre d'infections acquises au travail par 100000 personnes, médiane (ICr à $95 \%$ )

Nombre d'infections acquises dans des lieux publics, médiane (ICr à $95 \%$ )

Nombre d'infections acquises à domicile par 100000 personnes (ICr à $95 \%$ )

\section{Maintien de \\ l'éloignement sanitaire}

\section{Mesures minimales}

$$
\begin{gathered}
54,7(0,02-57,2) \\
33,5(0,02-35,1) \\
21,1(0,0-22,1) \\
38,5(16,7-42,6) \\
89,0(78,0-93,2) \\
11,0(6,8-22,0) \\
2,8(0,0-6,2) \\
25,6(0,0-50,0) \\
3,2(0,0-4,0)
\end{gathered}
$$

$54668(24-57177)$

$33539(20-35083$

$21082(4-22064)$

3652 (5-3979)

$930(0-996)$

$1088(0-1203)$

$8,0(0,0-9,2)$

$14,4(5,6-15,6)$

$25,6(16,8-32,8)$

$51,8(51,0-64,0)$

4199 (0-5169)

$7943(1-8340)$

$14190(7-14618)$

28280 (10-29 259)

$41,0(0,04-42,6)$
$25,1(0,02-26,2)$
$15,8(0,01-16,7)$
$38,7(31,5-43,9)$

$89,5(80,0-91,4)$

$10,5(8,6-20,0)$

$$
2,7(0,0-8,7)
$$$$
25,5(0,0-66,7)
$$

$$
2,9(0,0-4,0)
$$

\section{3 (35-42 579)}

$25062(23-26$ 158)

$118(22-8811)$

15955 (12-16 661)

2490 (3-2835)

$658(0-727)$

712 (0-790)

$8,5(0,0-9,4)$

$13,6(5,6-20,6)$

$24,2(19,4-31,0)$

$53,5(52,9-72,2)$

3449 (0-3874)

$5611(2-5930)$

$9924(7-10450)$

21889 (17-22 795)

$0,2(0,04-13,7)$

$0,1(0,02-8,8)$

$10,2(5,8-18,8)$

$2,8(0,9-12,5)$

$29,3(9,1-66,7)$

4,4 (1,2-10,0)

$13,4(5,5-20,0)$

$12(2-2114)$

$43(6-3008)$

$63(10-5286)$

\section{Dépistage et \\ recherche de contacts accrus}

Mesures combinées

$0,3(0,05-22,5)$

$0,3(0,03-1,0)$

$0,19(0,0-0,6)$

$0,11(0,01-0,4)$

$38,1(28,6-43,5)$

$37,3(25,0-44,5)$

$89,8(81,2-94,2)$

89,9 (81,2-94,7)

$10,1(5,3-18,8)$

$2,4(0,0-6,9)$

$22,5(0,0-50,0)$

$1,4(0,0-4,0)$

297 (28-990)

$191(18-625)$

$110(8-365)$

19 (2-67)

$4(0-17)$

$3(0-12)$

3,3 (0,0--12,3)

$13,9(0,0-20,6)$

$23,3(14,7-32,1)$

$23,6(15,4-31,8)$

$59,7(51,1-64,5)$

$58,9(51,5-68,2)$

$10(0-40)$

$44(0-124)$

$68(5-254)$

186 (32-12 074)

$167(15-580)$

Remarque : $\mathrm{ICr}=$ intervalle de crédibilité.

*Le tableau donne la médiane pour 50 simulations et les valeurs du 2,5e centile et du 97,5e centile, qui délimitent l'ICr à $95 \%$. Un ICr important indique un résultat dichotomique entre les simulations (p. ex. poursuite ou fin de l'épidémie). La médiane correspond au résultat le plus probable des 50 simulations.

établissements scolaires de 935 par 100000 personnes avec des mesures minimales et de 310 par 100000 personnes avec le maintien de l'éloignement sanitaire, par rapport aux scénarios seuls, mais son influence sur l'épidémie serait minime en raison de la poursuite de la transmission communautaire. La fermeture des écoles ferait augmenter la transmission à domicile (379 et
287 infections supplémentaires par 100000 personnes, respectivement), ce qui limite l'avantage de la transmission réduite dans les écoles. Dans le scénario de dépistage et de recherche de contacts accrus et le scénario des mesures combinées, la prolongation de la fermeture des écoles assure le contrôle de l'épidémie dans la plupart des simulations (figure 5). 


\section{A}

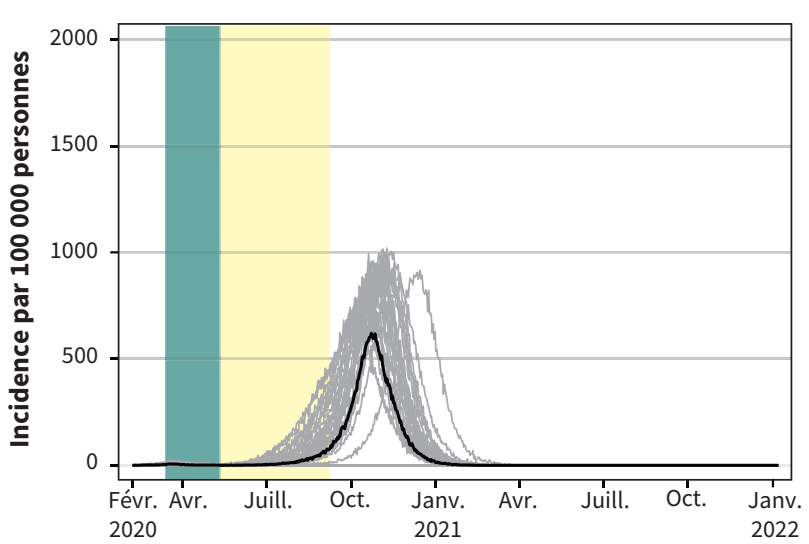

B

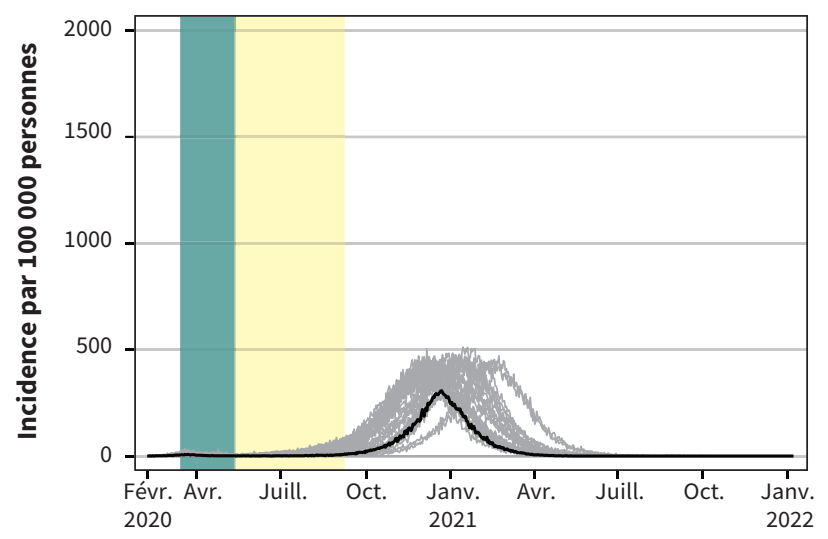

D

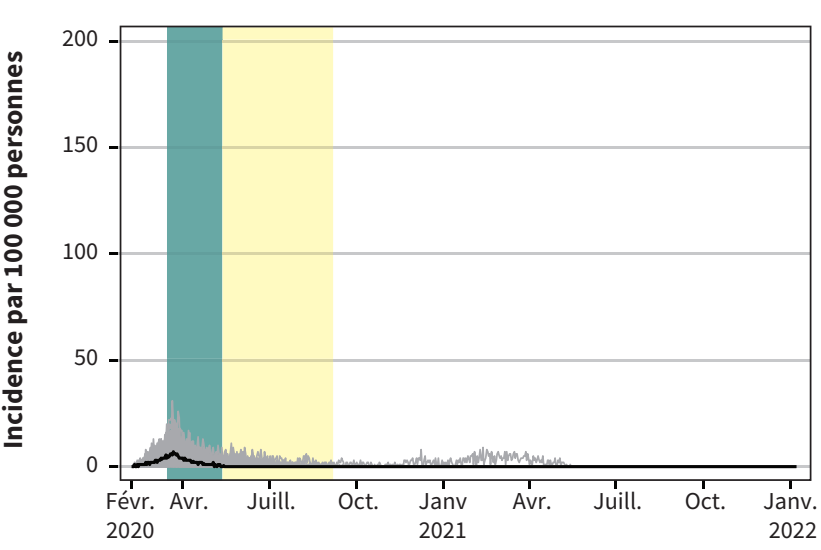

C

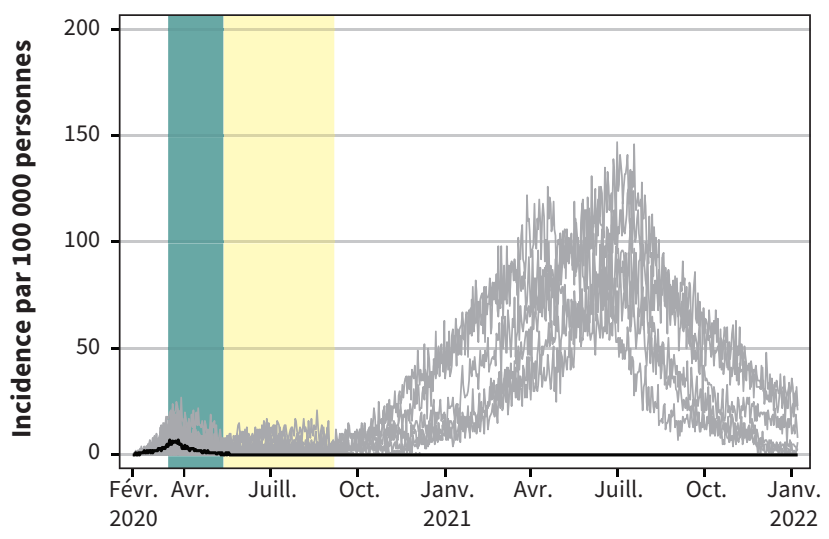

Dépistage et recherche de contacts accrus

igure 5: Courbes épidémiques projetées montrant le nombre quotidien de cas par 100000 personnes pour les 4 scénarios si l'on prolongeait la fermeture des écoles. Remarque: Dans chaque scénario, les mesures de santé publique s'intensifient graduellement. La bande verte représente la période de fermeture des espaces partagés, du 16 mars au 10 mai 2020. La bande jaune représente la période de fermeture des écoles sans la prolongation, du 11 mai au 8 septembre 2020. La ligne noire représente la médiane. Chaque ligne grise représente 1 simulation; il y en a 50 par scénario. L'échelle de l'axe des y est 10 fois plus petite pour le scénario de dépistage et de recherche de contacts accrus et pour le scénario des mesures combinées que pour les 2 autres scénarios.

En comparaison, la prolongation de la fermeture des lieux de travail et des lieux publics donne des taux d'attaque global beaucoup moins élevés avec les mesures minimales (0,4\%; ICr à $95 \%$ $0,1 \%-57,0 \%)$ et avec le maintien de l'éloignement sanitaire $(0,3 \%$; ICr à $95 \%$ 0,04\%-43,3\%) (tableau 5). L'épidémie prend fin dans plus de la moitié des simulations de prolongation selon ces scénarios, contre toutes les simulations pour le dépistage et la recherche de contacts accrus ou les mesures combinées (figure 6). Si la fermeture partielle des lieux de travail et des lieux publics est plus efficace que la fermeture des écoles, c'est parce qu'une plus grande proportion des infections sont acquises en milieu de travail (12,4\%-14,4\%) et dans les lieux publics $(22,5 \%-25,6 \%)$ que dans les écoles (3,3\%-9,0\%), quel que soit le scénario. La différence s'explique par le fait qu'il y a beaucoup plus de gens sur le marché du travail et dans la communauté qu'à l'école et par la poursuite de la transmission communautaire et à domicile malgré la fermeture des écoles.
Selon l'analyse de sensibilité, les résultats du modèle dépendent du paramètre de transmission ( $\beta$ ) et du taux de reproduction $\left(R_{0}\right)$ associé. Lorsque le $R_{0}$ est abaissé à 2,4 (comparativement à l'estimation pour la situation de référence, $\left.R_{0}=2,7\right)$, toutes les simulations des scénarios de dépistage et de recherche de contacts accrus et des mesures combinées montrent la fin de l'épidémie et le maintien de la demande sous la capacité des hôpitaux et des services de soins intensifs. Lorsque le $R_{0}$ est abaissé à 2,0, la capacité des hôpitaux et des unités de soins intensifs est aussi respectée pour le scénario du maintien de l'éloignement sanitaire, et même dans la plupart des simulations du scénario des mesures minimales. Au contraire, lorsque le $R_{0}$ est augmenté à 3,0 , aucun scénario ne permet le contrôle de l'épidémie, et seul le scénario des mesures combinées ne fait pas déborder les hôpitaux et les unités de soins intensifs (annexe 1, figures S13-S24). 
Tableau 5 : Résumé des résultats de la modélisation pour les 4 scénarios à l'étude, avec prolongation de la fermeture des espaces partagés (lieux de travail et lieux publics)*

\begin{tabular}{|c|c|c|c|c|}
\hline Variable & $\begin{array}{l}\text { Mesures } \\
\text { minimales }\end{array}$ & $\begin{array}{l}\text { Maintien de } \\
\text { l'éloignement } \\
\text { sanitaire }\end{array}$ & $\begin{array}{l}\text { Dépistage et } \\
\text { recherche de } \\
\text { contacts accrus }\end{array}$ & $\begin{array}{l}\text { Mesures } \\
\text { combinées }\end{array}$ \\
\hline Taux d'attaque global, \% (ICr à $95 \%)$ & $0,4(0,1-57,0)$ & $0,3(0,04-43,3)$ & $0,2(0,03-0,9)$ & $0,2(0,07-0,9)$ \\
\hline Taux d'attaque clinique, \% (ICr à $95 \%$ ) & $0,3(0,06-34,9)$ & $0,2(0,03-26,5)$ & $0,1(0,02-0,6)$ & $0,2(0,05-0,5)$ \\
\hline Taux de cas asymptomatiques, $\%$ (ICr à $95 \%)$ & $0,2(0,03-22,2)$ & $0,1(0,01-16,8)$ & $0,1(0,01-0,3)$ & $0,1(0,02-0,3)$ \\
\hline $\begin{array}{l}\text { Proportion de cas asymptomatique sur le nombre } \\
\text { total de cas, \% (ICr à } 95 \%)\end{array}$ & $38,5(33,8-42,6)$ & $38,8(28,0-43,6)$ & $37,9(25,3-44,7)$ & $37,5(27,4-44,3)$ \\
\hline Cas cliniques légers (pas hospitalisés), \% (ICr à $95 \%)$ & $89,0(85,5-93,1)$ & $89,6(83,3-93,8)$ & $89,5(82,2-93,2)$ & $89,6(79,7-93,1)$ \\
\hline $\begin{array}{l}\text { Cas cliniques hospitalisés (y compris aux soins } \\
\text { intensifs), \% (ICr à 95\%) }\end{array}$ & $11,0(6,9-14,5)$ & $10,4(6,2-16,7)$ & $10,5(6,8-17,8)$ & $10,4(6,9-20,3)$ \\
\hline Cas cliniques admis aux soins intensifs, \% (ICr à 95 \%) & $2,8(0,7-5,2)$ & $2,6(0,0-42,9)$ & $2,7(0,0-7,4)$ & $2,8(0,4-6,1)$ \\
\hline $\begin{array}{l}\text { Cas hospitalisés admis aux soins intensifs, } \\
\%(\mathrm{ICr} \text { à } 95 \%)\end{array}$ & $25,6(5,3-57,1)$ & $24,7(0,0-42,9)$ & $25,9(0,0-55,6)$ & $23,8(4,0-46,7)$ \\
\hline $\begin{array}{l}\text { Taux de mortalité parmi les cas cliniques, \% (ICr à } \\
95 \%)\end{array}$ & $3,2(0,0-4,7)$ & $1,7(0,0-4,8)$ & $1,4(0,0-4,3)$ & $1,5(0,0-5,1)$ \\
\hline $\begin{array}{l}\text { Total des cas (cliniques et asymptomatiques) par } \\
100000 \text { personnes, médiane (ICr à } 95 \%)\end{array}$ & $436(96-56988)$ & $290(42-43333)$ & $237(30-886)$ & $226(70-869)$ \\
\hline $\begin{array}{l}\text { Total des cas cliniques par } 100000 \text { personnes, } \\
\text { médiane (ICr à } 95 \%)\end{array}$ & $263(58-34923)$ & $169(29-26531)$ & $143(22-549)$ & $145(46-543)$ \\
\hline $\begin{array}{l}\text { Total des cas asymptomatiques par } \\
100000 \text { personnes, médiane (ICr à } 95 \%)\end{array}$ & $166(34-22$ 192) & $119(13-16802)$ & $89(7-337)$ & $91(22-326)$ \\
\hline $\begin{array}{l}\text { Total des cas hospitalisés par } 100000 \text { personnes, } \\
\text { médiane (ICr à 95\%) }\end{array}$ & $27(4-3922)$ & $18(5-2758)$ & $16(2-54)$ & $15(5-60)$ \\
\hline $\begin{array}{l}\text { Total des cas admis aux soins intensifs par } \\
100000 \text { personnes, médiane ( } \mathrm{ICr} \text { à } 95 \%)\end{array}$ & $11(1-1019)$ & $5(0-705)$ & $5(0-15)$ & $4(1-16)$ \\
\hline $\begin{array}{l}\text { Total des décès par } 100000 \text { personnes, médiane (ICr à } \\
95 \%)\end{array}$ & $5(0-1218)$ & $3(0-784)$ & $2(0-12)$ & $2(0-9)$ \\
\hline Infections acquises à l'école, \% (ICr à 95 \%) & $9,0(1,0-11,6)$ & $4,2(0,0-9,4)$ & $4,0(0,0-8,2)$ & $4,0(0,0-8,7)$ \\
\hline Infections acquises au travail, \% (ICr à $95 \%)$ & $14,2(3,8-20,5)$ & $13,6(8,3-18,4)$ & $12,4(5,3-18,9)$ & $12,5(7,1-23,0)$ \\
\hline $\begin{array}{l}\text { Infections acquises dans des lieux publics, \% (ICr à } \\
95 \%)\end{array}$ & $25,3(13,1-33,9)$ & $22,8(16,3-28,4)$ & $22,5(12,2-29,1)$ & $22,5(16,2-30,5)$ \\
\hline Infections acquises à domicile, \% (ICr à 95 \%) & $54,7(50,9-62,7)$ & $59,6(53,0-67,1)$ & $60,2(56,7-75,0)$ & $59,8(52,2-64,1)$ \\
\hline $\begin{array}{l}\text { Nombre d'infections acquises à l'école par } \\
100000 \text { personnes, médiane (ICr à } 95 \% \text { ) }\end{array}$ & $29(1-5307)$ & $11(0-3900)$ & $9(0-53)$ & $10(0-60)$ \\
\hline $\begin{array}{l}\text { Nombre d'infections acquises au travail par } \\
100000 \text { personnes, médiane (ICr à } 95 \%)\end{array}$ & $62(5-8194)$ & $41,5(5-5948)$ & $30(1-105)$ & $31(6-125)$ \\
\hline $\begin{array}{l}\text { Nombre d'infections acquises dans des lieux publics, } \\
\text { médiane (ICr à 95\%) }\end{array}$ & $109(17-14513)$ & $60(8-10461)$ & $52(4-201)$ & $47(14-199)$ \\
\hline Nombre d'infections acquises à domicile par & $243(52-29116)$ & $173(22-22991)$ & $138(15-541)$ & $139(35-508)$ \\
\hline
\end{tabular}

Remarque : $\mathrm{ICr}=$ intervalle de crédibilité.

*Le tableau donne la médiane pour 50 simulations et les valeurs du $2,5^{\mathrm{e}}$ centile et du $97,5^{\mathrm{e}}$ centile, qui délimitent l'ICr à $95 \%$. Un ICr important indique un résultat dichotomique entre les simulations (p. ex. poursuite ou fin de l'épidémie). La médiane correspond au résultat le plus probable des 50 simulations.

\section{Interprétation}

L'objectif était d'estimer la situation de la transmission du SRASCoV-2 au pays et de modéliser les défis auxquels nous devrons probablement faire face à la réouverture des espaces partagés au Canada. Malgré leur efficacité, les fermetures ont eu des effets néfastes sur le plan économique, sociale et de la santé. Le moment de la réouverture dépendra largement de la situation dans les provinces et territoires ${ }^{13,26}$. Notre modèle à base d'agents montre que toutes les mesures étudiées permettraient de réduire la transmission à un certain degré, mais que l'accroissement du dépistage pour isoler $50 \%$ des cas et 
A

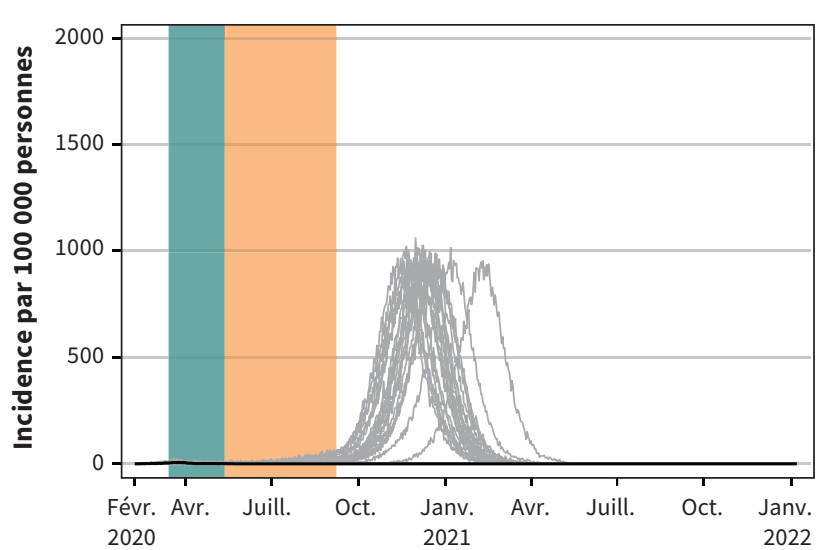

C

Dépistage et recherche de contacts accrus

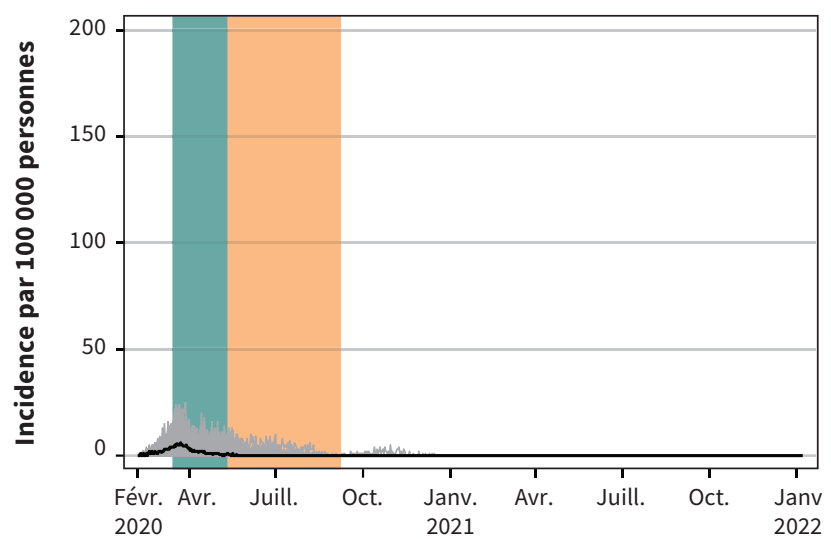

B

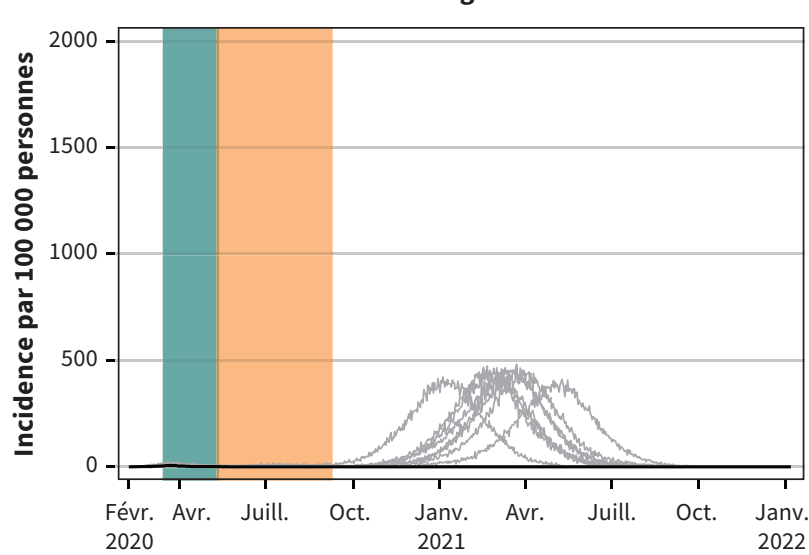

D
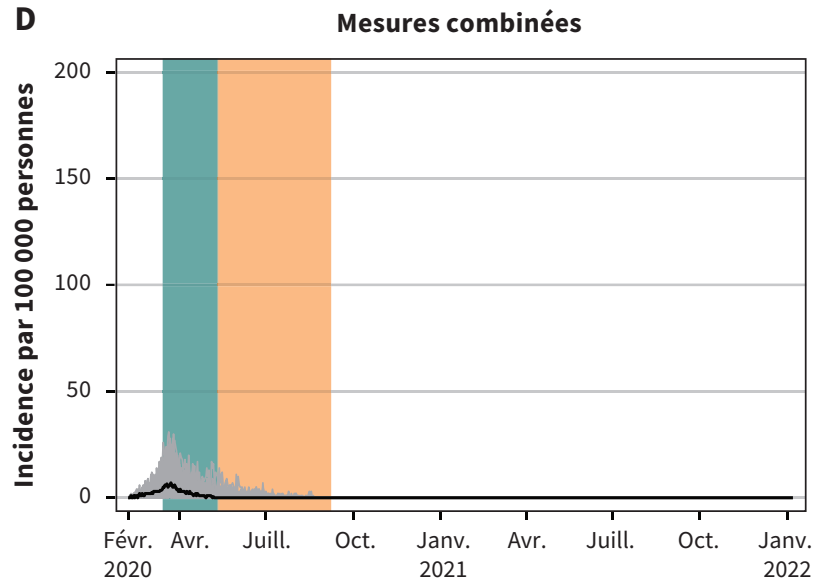

Figure 6: Courbes épidémiques projetées montrant le nombre quotidien de cas par 100000 personnes pour les 4 scénarios si l'on prolongeait la fermeture des écoles. Remarque : Dans chaque scénario, les mesures de santé publique s'intensifient graduellement. La bande verte représente la période de fermeture des espaces partagés, du 16 mars au 10 mai 2020. La bande orange représente la période de fermeture des écoles sans la prolongation, du 11 mai au 8 septembre 2020. La ligne noire représente la médiane. Chaque ligne grise représente 1 simulation; il y en a 50 par scénario. L'échelle de l'axe des y est 10 fois plus petite pour le scénario de dépistage et de recherche de contacts accrus et pour le scénario des mesures combinées que pour les 2 autres scénarios.

l'intensification de la recherche visant à mettre en quarantaine tous les contacts de ces cas constituent la méthode la plus efficace, surtout lorsqu'on la combine avec le maintien de l'éloignement sanitaire qui réduirait de $20 \%$ le taux de contacts dans la population. Ces mesures devront rester en place jusqu'à ce que l'épidémie prenne fin (grâce à l'immunité collective ou à la vaccination), sans quoi il y aura une résurgence des cas.

La fermeture partielle des espaces partagés est la seule mesure étudiée qui permettrait à elle seule d'endiguer l'épidémie. Les résultats portent à croire que c'est principalement la fermeture des lieux de travail et des lieux publics, et non des écoles, qui serait efficace. La fermeture des écoles, bien qu'elle puisse prévenir la transmission en milieu scolaire, n'augmente que peu l'effet de la fermeture partielle des espaces partagés et ne suffit pas pour réduire le taux d'attaque global et contrôler l'épidémie, surtout si la transmission communautaire - majoritairement due aux personnes asymptomatiques et présymptomatiques contagieuses - persiste. Sans autres mesures de santé publique, elle ne ferait que prolonger l'épidémie ${ }^{14}$. Nos conclusions concordent avec celles des premières études, qui indiquaient que la fermeture des écoles pourrait ne pas être aussi efficace que d'autres mesures moins restrictives ${ }^{27}$. La fermeture des lieux de travail et des espaces partagés se révèle beaucoup plus efficace, parce que la transmission se fait principalement dans ces endroits. Comme des études examinant la situation d'autres pays ${ }^{14,28}$, nous avons conclu qu'en l'absence de mesures, environ les deux tiers des Canadiens seraient infectés. Nous avons fait des projections pour des mesures réalistes et cohérentes avec les directives en vigueur au Canada afin de réduire la proportion de la population infectée, qui diminuera quelque peu ou presque entièrement selon la trajectoire des mesures prises dans les prochains mois, tel que démontré dans d'autres modèles ${ }^{14,16}$. Le taux d'attaque réel dépendra du degré d'accroissement du dépistage et de l'isolement ainsi que de la recherche de contacts et de la mise en quarantaine ${ }^{21,29}$, et de la mesure dans laquelle la population canadienne adopte les 
mesures d'éloignement sanitaire et de protection individuelle $22,24,30$. Si nous rouvrons les espaces partagés sans mettre en place d'autres mesures de santé publique, l'épidémie reprendra dans une ampleur tributaire de nos efforts à venir, ce qui fera déborder le système de santé et obliger les autorités à réinstaurer des mesures de confinement.

\section{Limites de l'étude}

De nombreuses caractéristiques épidémiologiques importantes du SRAS-CoV-2 demeurent inconnues, particulièrement la susceptibilité (possiblement inférieure) des enfants à l'infection par rapport aux adultes, le taux réel d'infections asymptomatiques et l'apparition d'une immunité durable. Nous avons créé un modèle national qui tente d'agréger des épidémies régionales hétérogènes; il pourrait donc ne pas être représentatif de la transmission du SRAS-CoV-2 dans toutes les collectivités. Néanmoins, nous avons calibré le modèle à partir de données canadiennes, et les résultats montrent une bonne correspondance avec les cas acquis localement. Notre modèle ne tient pas compte de la transmission parmi les travailleurs de la santé et les résidents des établissements de soins de longue durée, qui sont la source de multiples éclosions au pays. Comme la transmission dans ces 2 groupes n'a pas la même ampleur dans toutes les provinces et tous les territoires, un modèle national ne permet pas d'étudier ces grappes à l'échelle régionale et locale. De plus, les milieux où surviennent les éclosions sont confrontés à des enjeux uniques qui exigent souvent des mesures de contrôle et de prévention des infections plus intenses que celles mises en place dans la population générale ${ }^{31}$. Notre modèle est représentatif de la situation nationale et donne une projection de référence de la transmission communautaire au Canada. C'est pourquoi il faut interpréter les résultats en tenant compte que, dans certaines circonstances, des éclosions locales pourraient mener à un nombre de cas, d'hospitalisations et de décès plus élevé que prévu.

\section{Conclusion}

Le modèle fournit des estimations provisoires de la transmission de la COVID-19 dans la population canadienne et des effets des mesures combinées en matière de réduction et d'élimination de la transmission au pays. Les premières mesures de contrôle de l'épidémie ont été très efficaces, mais lorsque les mesures restrictives seront levées, il faudra intensifier d'autres mesures pour prévenir la transmission, réduire au minimum le nombre de cas et ne pas surcharger les systèmes de santé.

\section{Références}

1. Allocution liminaire du Directeur général de l'OMS lors du point de presse sur la COVID-19 - 11 mars 2020. Genève : Organisation mondiale de la Santé 2020. Accessible ici : https://www.who.int/fr/dg/speeches/detail/who-director-general -s-opening-remarks-at-the-media-briefing-on-covid-19---11-march-2020 (consulté le 2 mai 2020)

2. Coronavirus disease 2019 (COVID-19): situation report, 51. Geneva: World Health Organization; 2020:1-9.

3. COVID-19 Dashboard by the Center for Systems Science and Engineering (CSSE) at Johns Hopkins University. Baltimore : Université John Hopkins. Accessible ici : https://gisanddata.maps.arcgis.com/apps/opsdashboard/ index.html\#/bda7594740fd40299423467b48e9ecf6 (consulté le 28 juin 2020).
4. Wu Z, McGoogan JM. Characteristics of and important lessons from the coronavirus disease 2019 (COVID-19) outbreak in China: summary of a report of 72314 cases from the Chinese Center for Disease Control and Prevention. JAMA 2020;323:1239-42. doi : 10.1001/jama.2020.2648.

5. Garg S, Kim L, Whitaker M, et al. Hospitalization rates and characteristics of patients hospitalized with laboratory-confirmed coronavirus disease 2019 COVID-NET, 14 states, March 1-30, 2020. MMWR Morb Mortal Wkly Rep 2020;69: 458-64.

6. Wang D, Hu B, Hu C, et al. Clinical characteristics of 138 hospitalized patients with 2019 novel coronavirus-infected pneumonia in Wuhan, China. JAMA 2020;323:1061-9.

7. Hu Z, Song C, Xu C, et al. Clinical characteristics of 24 asymptomatic infections with COVID-19 screened among close contacts in Nanjing, China. Sci China Life Sci 2020;63:706-11.

8. Xia W, Liao J, Li C, et al. Transmission of corona virus disease 2019 during the incubation period may lead to a quarantine loophole. medRxiv 8 mars 2020. doi : 10.1101/2020.03.06.20031955.

9. Tong Z-D, Tang A, Li K-F, et al. Potential presymptomatic transmission of SARS-CoV-2, Zhejiang Province, China, 2020. Emerg Infect Dis 2020;26:1052-4.

10. Wei WE, Li Z, Chiew CJ, et al. Presymptomatic transmission of SARS-CoV-2 Singapore, January 23-March 16, 2020. MMWR Morb Mortal Wkly Rep 2020;69: 411-5.

11. Silverstein WK, Stroud L, Cleghorn GE, et al. First imported case of 2019 novel coronavirus in Canada, presenting as mild pneumonia. Lancet 2020;395:734.

12. Maladie à coronavirus de 2019 (COVID-19) : Mise à jour quotidienne sur l'épidémiologie. Ottawa : Agence de la santé publique du Canada. Modifié le 20 juillet 2020. Accessible ici : https://sante-infobase.canada.ca/covid-19/resume -epidemiologique-cas-covid-19.html (consulté le 10 juin 2020).

13. Federal/provincial/territorial COVID-19 interventions [document interne]. Ottawa : Santé Canada. 4 mai 2020. Accessible sur demande auprès de l'auteur.

14. Ferguson N, Laydon D, Nedjati-Gilani G, et al. Report 9: Impact of non-pharmaceutical interventions (NPIs) to reduce COVID-19 mortality and healthcare demand. London (UK) : Imperial College London; 2020.

15. Hellewell J, Abbott S, Gimma A, et al. Feasibility of controlling COVID-19 outbreaks by isolation of cases and contacts. Lancet Glob Health. 2020;8:e488-96.

16. Tuite AR, Fisman DN, Greer AL. Mathematical modelling of COVID-19 transmission and mitigation strategies in the population of Ontario, Canada. CMAJ 2020;192:E497-505.

17. Kermack W, McKendrick A. A contribution to the mathematical theory of epidemics. Proc R Soc Lond, A Contain Pap Math Phys Character 1927;115:700-21. doi: 10.1098/rspa.1927.0118.

18. Althaus CL. Estimating the reproduction number of Ebola virus (EBOV) during the 2014 outbreak in West Africa. PLoS Curr. 2014;6. doi : 10.1371/currents.outb reaks.91afb5e0f279e7f29e7056095255b288.

19. Chao DL, Oron AP, Srikrishna D, et al. Modeling layered non-pharmaceutical interventions against SARS-CoV-2 in the United States with Corvid. medRxiv 11 avril 2020. doi : 10.1101/2020.04.08.20058487.

20. Davies NG, Kucharski AJ, Eggo RM, et al. Centre for the Mathematical Modelling of Infectious Diseases COVID-19 working group. Effects of non-pharmaceutical interventions on COVID-19 cases, deaths, and demand for hospital services in the UK: a modelling study. Lancet Public Health 2020;5:e375-85.

21. Using a delay-adjusted case fatality ratio to estimate under-reporting. Londres (Royaume-Uni) : Centre for Mathematical Modeling of Infectious Diseases, London School of Hygiene \& Tropical Medicine. 22 mars 2020, modifié le 21 juin 2020. Accessible ici : https://cmmid.github.io/topics/covid19/global_cfr_estimates.html (consulté le 21 juin 2020).

22. COVID-19 Community Mobility Report. Google Canada; 2020. Accessible ici : www. gstatic.com/covid19/mobility/2020-05-09_CA_Mobility_Report_en.pdf (consulté le 9 mai 2020).

23. Série d'enquêtes sur les perspectives canadiennes 1 : La COVID-19 et travailler de la maison, 2020. Ottawa : Statistique Canada. Le Quotidien. 2020. Accessible ici : https://www150.statcan.gc.ca/n1/daily-quotidien/200417/dq200417a-fra. pdf (consulté le 17 avril 2020).

24. Comment les Canadiens vivent-ils la situation liée à la COVID-19? [infographie]. Ottawa : Statistique Canada. Modifié le 20 avril 2020. Accessible ici : https:// www150.statcan.gc.ca/n1/pub/11-627-m/11-627-m2020029-fra.htm (consulté le 19 avril 2020).

25. Tang B, Scarabel F, Bragazzi NL, et al. De-escalation by reversing the escalation with a stronger synergistic package of contact tracing, quarantine, isolation and personal protection: feasibility of preventing a COVID-19 rebound in Ontario, Canada, as a case study. Biology (Basel) 2020;9:100. 
26. COVID-19 Canada : Tableau de bord de l'état de la situation. Ottawa : Agence de la santé publique du Canada. Accessible ici : https://experience.arcgis.com/ experience/f16b85c700c94729a2cd1c6efe5f6839/ (consulté le 27 juin 2020).

27. Viner RM, Russell SJ, Croker H, et al. School closure and management practices during coronavirus outbreaks including COVID-19: a rapid systematic review. Lancet Child \& Adolesc Health 2020;4:397-404.

28. Kwok KO, Lai F, Wei WI, et al. Herd immunity - estimating the level required to halt the COVID-19 epidemics in affected countries. J Infect 2020;80:e32-3.

29. Scarabel F, Pellis L, Bragazzi NL, et al. Canada needs to rapidly escalate public health interventions for its COVID-19 mitigation strategies. Infect Dis Model 2020;5:316-22. doi : 10.1016/j.idm.2020.03.004.
30. Chu DK, Akl EA, Duda S, et al.; auteurs de l'étude COVID-19 Systematic Urgent Review Group Effort (SURGE). Physical distancing, face masks, and eye protection to prevent person-to-person transmission of SARS-CoV-2 and COVID-19: a systematic review and meta-analysis. Lancet 2020;395:1973-87.

31. Callaghan AW, Chard AN, Arnold P, et al. Screening for SARS-CoV-2 infection within a psychiatric hospital and considerations for limiting transmission within residential psychiatric facilities - Wyoming, 2020. MMWR Morb Mortal Wkly Rep 2020;69:825-9.

\section{Intérêts concurrents : Aucun déclaré.}

Cet article a été révisé par des pairs.

Affiliations : Division des sciences des risques pour la santé publique (Ng, Fazil, Waddell, Turgeon, Otten, Ogden), Laboratoire national de microbiologie, Direction générale de la prévention et du contrôle des maladies infectieuses, Agence de la santé publique du Canada, Guelph (Ontario) et Saint-Hyacinthe (Québec); Centre de l'immunisation et des maladies respiratoires infectieuses (Bancej), Direction générale de la prévention et du contrôle des maladies infectieuses, Agence de la santé publique du Canada, Ottawa (Ontario); Bureau des programmes et de la planification en biosécurité (Atchessi), Centre de la biosûreté, Direction générale de l'infrastructure de sûreté sanitaire, Agence de la santé publique du Canada, Ottawa (Ontario).

Collaborateurs : Victoria Ng, Aamir Fazil et Nicholas Ogden ont contribué à la conceptualisation. Ainsley Otten, Lisa Waddell et Nicole Atchessi ont contribué à la collecte de données. Victoria Ng a contribué à l'analyse. Christina Bancej et Patricia Turgeon ont contribué à l'interprétation des données. Victoria Ng, Christina Bancej et Patricia
Turgeon ont rédigé l'ébauche de l'article, que tous les auteurs ont révisé. Tous les auteurs ont donné leur approbation finale pour la version soumise pour publication et assument l'entière responsabilité de tous les aspects du travail.

Partage des données : Toutes les données issues des modélisations peuvent être obtenues sur demande auprès de l'auteure de correspondance, tout comme les données épidémiologiques utilisées. Les données liées au nombre de cas de COVID-19, au nombre d'hospitalisations et à la mortalité seront sujettes aux lois sur la confidentialité. Les personnes intéressées peuvent communiquer avec l'auteure de correspondance, qui les mettra en contact avec la source des données d'origine.

Remerciements : Les auteurs remercient Lujie Duan de l'Université de la Saskatchewan, André Jacques de SimWell Consulting and Technologies et Deirdre Hennessy de Statistique Canada pour leur aide sur le plan technique.

Accepté : 21 juillet 2020

Correspondance : Victoria Ng, victoria.ng@canada.ca 